\title{
Demonstrating the Practical Challenges of Wireless Communications Using USRP
}

\author{
Mohammed El-Hajjar, Quoc A. Nguyen, Robert G. Maunder, and Soon Xin Ng
}

\begin{abstract}
Wireless communications is taught in most electronics degree curricula worldwide, where the main focus is the theory of communications. While it is essential that students learn the theory of communications, this is not enough to prepare them for their careers in industry. This is due to the fact that there are several practical challenges in designing and implementing wireless communications systems that are overlooked when focusing only on theory. Hence, in this article we describe the main challenges in designing practical communications systems, and demonstrate how the National Instruments Universal Software Radio Peripheral can be used for introducing and educating students about these challenges.
\end{abstract}

\section{INTRODUCTION}

Digital communications is taught in most electrical and electronics degree curricula worldwide, where students normally learn the theory of communications and understand the different building blocks of a communications system. However, learning the theory alone does not prepare students for the marketplace as there are several challenges in designing communications systems, including, for example, channel estimation and synchronization. Students mostly use simulations in order to design and characterize communications systems, where these simulations normally depend on simplifying impractical assumptions. Therefore, software defined radio (SDR) can be utilized in order to reinforce the theory with hands-on learning. An SDR is a communications platform that uses software for implementing digital communications algorithms.

In our course at the University of Southamp-

Mohammed El-Hajjar, Quoc A. Nguyen, Robert G. Maunder, and Soon Xin Ng are with the University of Southampton.

The financial support of the EPSRC, Swindon UK under the grant EP/J015520/1 is gratefully acknowledged. learn some useful tools and gaine experience in designing, implementing, and testing communications systems.

This article presents the experience of using the USRP in order to teach students hands-on learning techniques for linking theory with practical implementation. Here we present two case studies, where we first present a laboratory session using the USRP and then describe a project using the USRP, and share the lessons learned during setup and while running the laboratory as well as when supervising the project.

In this article, we first outline the pedagogical motivation for using the NI USRP in our wireless communications courses. Then the USRP platform is described, followed by a description of two USRP-based projects. Finally, our reflections are given followed by our conclusions.

\section{Pedagogical Motivation}

Digital communications is taught in most electrical and electronics engineering undergraduate and postgraduate degree curricula worldwide, where these modules mostly focus on the theoretical aspects of communications systems, while theoretically studying the blocks of Fig. 1. More specifically, Fig. 1 shows a typical block diagram of a communications system, including the basic components of source coding/decoding, channel coding/decoding, modulation/demodulation, as well as the analog processing for transmission over the wireless channel.

As shown in Fig. 1, a typical wireless communications system will have a signal source, where source coding is applied to the signal in order to remove any redundancy in the source data [2]. After source coding, the data bits are channel coded as shown in Fig. 1, where channel coding is used for error detection and correction at the receiver. After channel coding, the data bits are mapped to symbols in the modulator, the output symbols of which are passed through an analog processing block before transmission in the channel [3]. After transmission in the channel, as shown in Fig. 1, the reverse processing of the transmitter is applied in the receiver.

Students should learn and understand these concepts theoretically; however, most courses tend to ignore the practical implementation aspects of communications systems. Students mostly validate the theory by Matlab or $\mathrm{C}$ simu- 
lations of baseband systems, normally constructed based on simplifying assumptions. Simulation of wireless communication systems using these simplifying assumptions means that most of the time students are not well prepared to apply their knowledge in a communications product. Hence, we decided to introduce a practical session in our courses, where we opted to use the NI USRP. This was intended to give students the chance to understand the main challenges when designing and implementing communications systems.

In what follows we list the main challenges in communications systems, which the students would be able to understand while using the USRP. ${ }^{1}$

Pulse shaping: The digital signal output of the modulator in Fig. 1 cannot be transmitted easily over the wireless channel and hence needs to be mapped to an analog waveform that can be. This involves mapping the complex symbols output of the modulators of Fig. 1 to an analog pulse train for transmission over the wireless channel. Then the receiver would perform an analogous operation to recover the complex symbols from the received pulses using a matched filter. Students normally learn about pulse shaping and the characteristics of a Nyquist system [3]; however, they would not fully understand its implications due to the lack of practical implementation in the curriculum. Additionally, students would understand the importance of upsampling and downsampling in a transceiver more when they need to design a filter, since upsampling is needed in order to allow for simpler filter implementation, where increasing the sampling frequency will relax the sharp cutoff requirements on the filters.

Bandwidth and data rate: Students normally study the importance of bandwidth in communications systems and its relation with the data rate via the Nyquist theorem. However, they do not learn how these can be used in practical systems and how they can be related to the processor speed running the receiver, for example.

Frame detection: The data is practically transmitted in frames with predefined structure, and it is essential for the decoder to know the beginning of a frame in order to be able to decode the signal. In practice, due to propagation and signal processing delays, the location of the beginning of the frame is unknown. Hence, detecting the beginning of a frame forms an integral part of any communications receiver, which can be considered as part of the receiver synchronization.

Timing recovery: When the signal is received, it is a complicated analog signal that should be sampled before recovering the digital data. Wireless channels introduce attenuation, phase shift, and time delay, which are unknown to the receiver, and the receiver needs to find the correct sampling point in order to be able to sample the received analog signal [4]. This aspect of wireless system design is often ignored in baseband simulation; hence, using NI USRP, students would learn the importance of this in communications products. On the other hand, an additional challenge includes the offset between the sampling rates at the transmitter

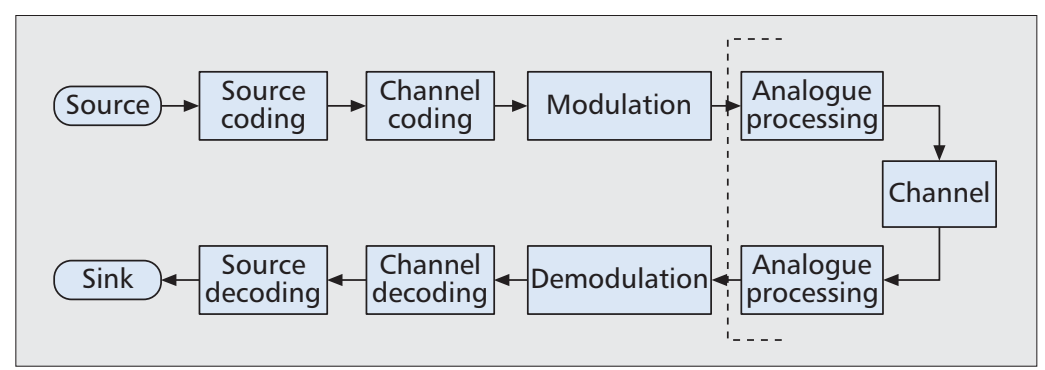

Figure 1. Typical communications system components.

and the receiver, which may also be variable. Hence, sampling frequency offset estimation as well as tracking the variation in this offset need to be considered when designing communications receivers.

Carrier recovery: Before sampling the received signal, the receiver down-converts the received analog signal to baseband, where the receiver must know the carrier frequency and phase of the received signal for successful demodulation. This is normally done via an oscillator, whose frequency is matched to the transmitter's carrier frequency. However, the receiver should estimate the phase and carrier frequency offsets introduced by the wireless channel and the imperfections in the circuitry [4]. Furthermore, the carrier frequency offset may vary with time; hence, tracking the carrier frequency offset is another important challenge for designing communications receivers.

Channel state information estimation: In order to perform correct equalization, coherent wireless receivers need to know the channel, which is normally estimated by the receiver. Hence, in order to facilitate channel estimation, pilot symbols, which are known at the receiver, are transmitted periodically. Additionally, random noise is normally added to the signal in the receiver, which is normally modeled as Gaussian noise [3]; and when soft-decoding [3] is employed, the receiver should also estimate the noise variance in order to be able to implement soft demodulation.

Finally, there are several other considerations when designing wireless systems, including upconversion at the transmitter and down-conversion at the receiver, sampling and quantization, input signal level measurement, automatic gain control (AGC), and common phase error cancellation.

In summary, wireless communication is now used in most aspects of our lives, and is taught in undergraduate and postgraduate electronics curricula with more focus on theory than application and implementation. Students mainly focus on simulations of communications systems making several simplifying assumptions, which mainly ignore most of the above-mentioned practical aspects of transceiver design. Hence, we aimed at introducing the USRP in our courses in order to teach digital communications from an implementation point of view in order to complement the theory.
${ }^{1}$ Note that the list includes the challenges that students would not experience when simulating baseband systems. 


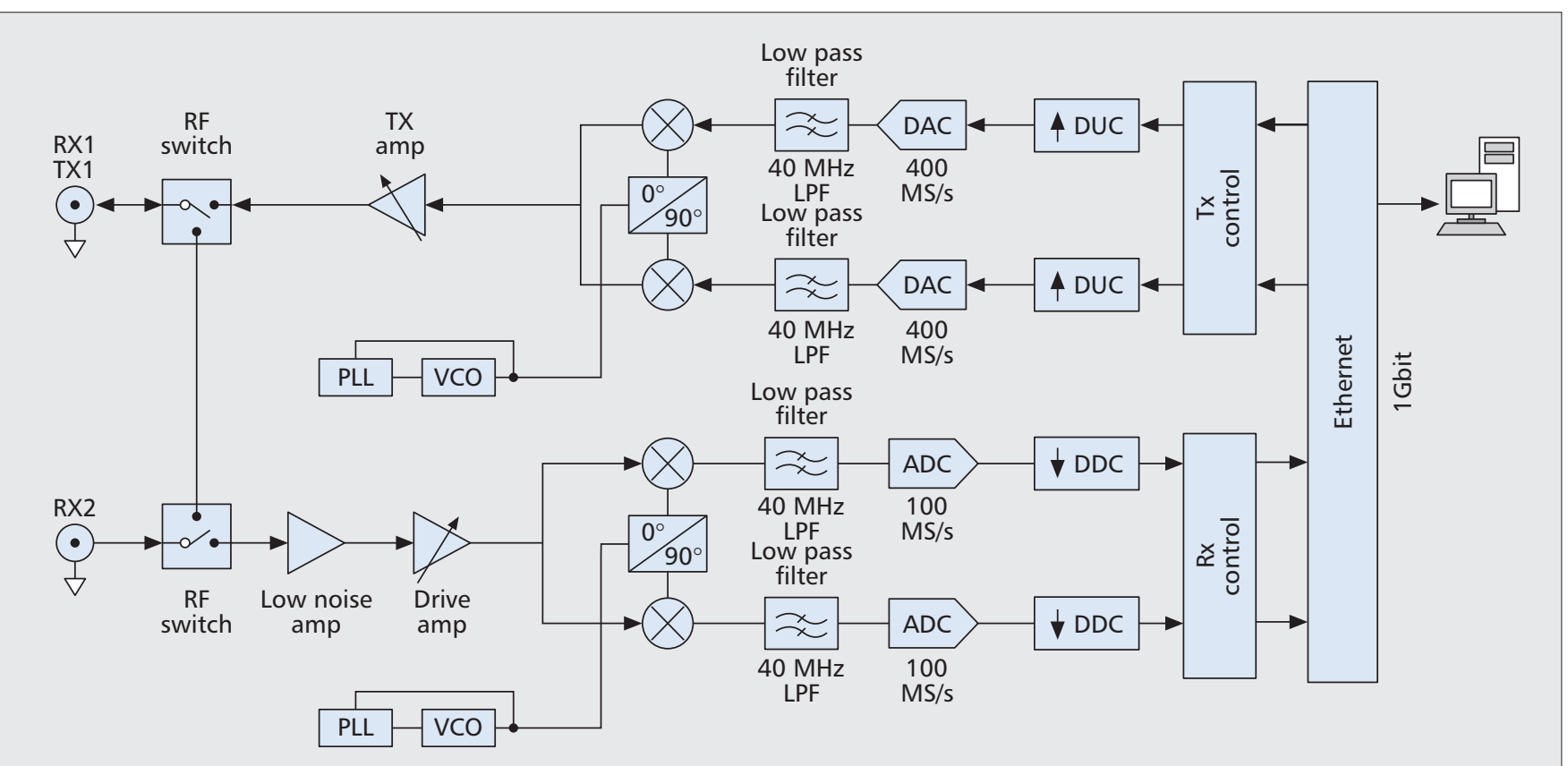

Figure 2. NI USRP-2920 system block diagram (c National Instruments.

\section{THE UNIVERSAL SoftWARe RADIo Peripheral}

The USRP is an SDR reconfigurable RF hardware designed to build and test digital communication systems. The USRP is commonly used by researchers as a wireless prototyping platform [5] and by universities as a teaching aid [6]. National Instruments has further developed a graphical programming software called Laboratory Virtual Instrument Engineering Workbench (LabVIEW), which is a system-design platform and development environment. With support from LabVIEW, real-time processing is made possible when data is streamed to and from the USRP via a gigabit-per-second Ethernet connection [6]. Hence, the availability of both USRP and LabVIEW has benefited various researchers and educators.

For example, when transmitting a phase shift keying (PSK)-modulated signal, the processing of the bits and mapping them to PSK symbols is done in LabVIEW. Afterward, the I/Q LabVIEW-processed signal is passed to the USRP over the gigabit Ethernet. The USRP upconverts the signal to RF according to the USRP configuration set up in LabVIEW. Then the USRP amplifies and transmits the signal over the air. The USRP-2920 used in our labs and projects supports frequencies in the range $50 \mathrm{MHz}-2.2$ $\mathrm{GHz}$ with up to $25 \mathrm{MHz}$ bandwidth, which covers the frequency range for applications including broadcast FM, public safety, land mobile, low-power unlicensed devices, sensor networks, cell phones, amateur radio, and GPS.

On the other hand, the NI USRP is also capable of receiving the signal when the received signal is mixed down from RF using a direct conversion receiver to baseband I/Q components. The digitized I/Q data follows parallel paths through a digital down-conversion process that mixes, filters, and decimates the input signal to a user-specified rate. The down-converted samples are then passed to the host computer over a standard gigabit Ethernet connection. Figure 2 shows a block diagram of the USRP for the transmit and receive channels. As shown in Fig. 2, the NI USRP-2920 platform is capable of transmitting and receiving using two antennas, which implies that it is possible to implement a twin-antenna multiple-input multiple-output (MIMO) system using this platform.

\section{USRP PRACTICAL SESSION AND EXAMPLE PROJECT}

\section{USRP Practical Session: Differential PSK}

The Department of Electronics and Computer Science at the University of Southampton runs a Master's course in wireless communications, which is a one-year intensive course with focus on mobile speech and data communications [7]. Students in this course study the theory of digital communications and learn about the different communications techniques using Matlab/C simulations. Hence, in order to introduce a more practical aspect to the course, a practical session was introduced using NI USRP, which was run for the first time during academic year 2012-2013.

Overview - This practical session aims at giving students the chance to construct a wireless communication link using the NI USRP in order to gain a better understanding of the communications theory and link it with real implementation. The aims of the practical session were set as:

- Understand the USRP hardware and capabilities;

- Build a differential quadrature PSK (DQPSK) receiver using LabVIEW and the USRP 


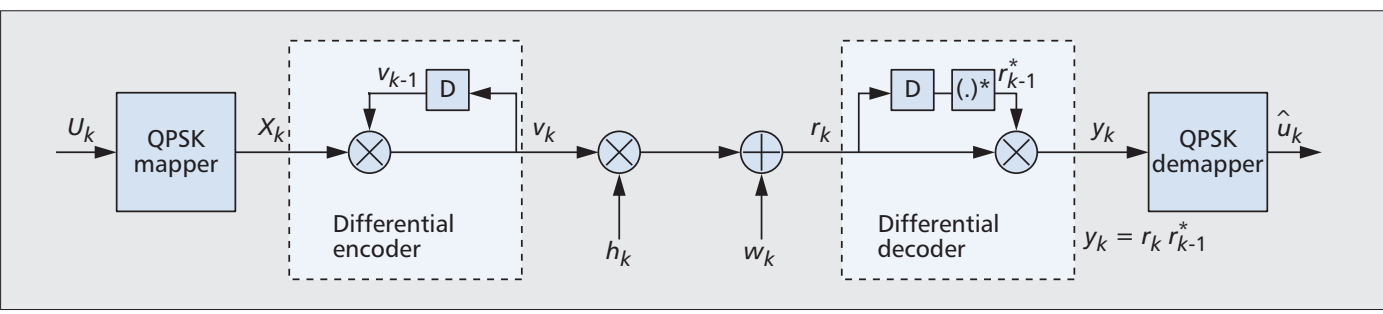

Figure 3. Baseband system block diagram of DQPSK transmission and detection.

Additionally, the practical session learning outcomes were set as:

- Understand how to build a communications system using NI USRP

- Appreciate the challenges in designing a communications system

- Link the theory with practical implementation

Before the practical session, the students were given a one-hour lecture on USRP and how they could achieve the laboratory's learning outcomes. Then they were given a pre-session assignment, where they would learn about LabVIEW and its functionalities. During the practical session, students worked in groups of two to build a DPSK transceiver in three hours. The pre-session documents can be downloaded from http:/www.edshare.soton.ac.uk/9681/, and the practical session material can be downloaded from http://www.edshare.soton.ac.uk/9682/ and http://www.edshare.soton.ac.uk/9683/.

Practical Challenges - The main purpose of differential modulation is to avoid the need for channel estimation during the demodulation of the received signal [8]. The baseband system block diagram of DQPSK transmission and detection is shown in Fig. 3. More specifically, the QPSK mapper maps two bits from the bit sequence $u_{k}$ to a complex-valued symbol $x_{k}$. Then the differential encoder encodes the QPSK symbol to a new complex-valued symbol as $v_{k}=$ $v_{k-1} \cdot x_{k}$ for transmission over the channel, as shown in Fig. 3. The received signal can be represented as $r_{k}=h_{k} \cdot v_{k}+w_{k}$, where $h_{k}=$ $\left|h_{k}\right| e^{\angle h k}$ and $w_{k}=\left|\mathrm{w}_{k}\right| e^{\angle n k}$ are the complexvalued channel fading and noise in the baseband. Note that the baseband channel $h_{k}$ is a simplified way of modeling the transmit and receive filters, modulation and demodulation, as well as the physical passband channel, based on various idealistic assumptions mentioned earlier.

DPSK is normally taught in class using the block diagram in Fig. 3. However, in practice, after the differential encoder, the DQPSK symbol sequence $v_{k}$ of Fig. 3 is upsampled and then passed to a root-raised cosine filter, as shown in the LabVIEW block diagram in Fig. 4. Afterward, the filtered signal is modulated and passed to the USRP hardware for transmission over the wireless channel. At the receiver, the received signal needs to be detected by the USRP and fed to LabVIEW for demodulation, filtering, and down-sampling, in order to obtain the baseband DQPSK signal $r_{k}$ as shown in Fig. 3 . The differentially decoded signal is given by $y_{k}=r_{k}$. $r_{k-1}^{*}$, which depends on the current and previous baseband DQPSK signals as shown in Fig. 3; hence, there is no need for channel estimation, which simplifies the detection process.

Technical Approach - In this practical session, the students were asked to build a DQPSK demodulator using LabVIEW. An incomplete DQPSK demodulator was provided to the students, who were asked to complete the decoder in order to decode a signal transmitted from another USRP. The LabVIEW front panels of the DQPSK signals constellation graph and eye diagram are shown in Fig. 5. The students were asked to complete this task based on the following procedures:

1. Apply the root raised cosine shaping filter to the received and down-converted signal, and test the output by comparing to the input of the filter in the transmitter.

2 Insert and connect a down-sampler into the demodulator, and test the output by comparing it to the input of the filter in the transmitter.

3 Implement the differential demodulation function, as shown in Fig. 3, in order to process the down-sampled signals and test the filter in the transmitter.

4 Insert and connect a PSK demodulation code into the demodulator followed by using the For Loop function palette in LabVIEW for converting the constellation points to symbols. Students were given stepby-step instructions for implementing For Loop.

5 Once that was done, students were asked to test their implementation as well as to compare their constellation graph and signal eye diagram at their receiver to those shown as the transmitter in Fig. 5.

6 Finally, the students were asked to record their observations after modifying various detection parameters, including carrier frequency, roll-off factor, and antenna gain.

Project Outcome - After the practical session, the students were asked to fill a feedback questionnaire, where they were asked about their satisfaction with the practical session and any extra information they would like to be added to the practical session. A total of 44 students completed the questionnaire, of which 36 students $(82$ percent) said that they would like to make more use of USRPs during the curriculum of their Master's studies, and 33 students (75 percent) said that they would like to use the USRPs as the basis of their Master's research projects. The following are some quotes from the student feedback: the output by comparing it to the input of
Before the practical

session, the students

were given a

one-hour lecture on

USRP and how they

can achieve the lab's

learning outcomes.

Then, they were

given a pre-session

assignment, where

they would learn

about the

LabVIEW and its

functionalities. 


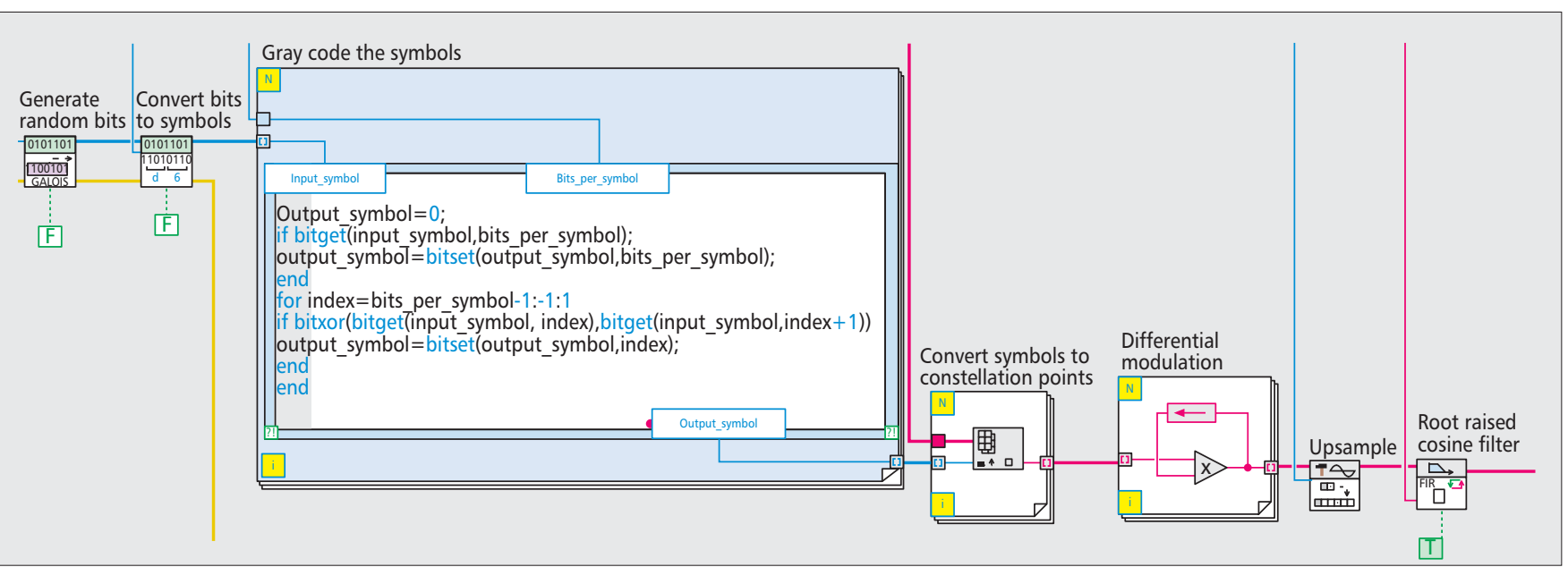

Figure 4. The LabVIEW block diagram of a DQPSK transmitter, including the random bit generator, bits-to-symbols converter, Gray coding, symbols-to-constellation-points converter, differential modulation, upsampling, and root raised cosine filter.

"The USRP has given me a passion for hardware implementation, which I didn't have before. I hope that I can get access to the USRPs any time."

"The USRP gives an avenue for exploration. It is a good tool to bridge the gap between practical and theory."

"The USRP vividly helps me understand the theory that I learned in class."

Final Year Project: Phase Shift Keying Using Pilot SYMbol Assisted Modulation

In this section, we discuss a project in which the USRP was employed to demonstrate PSK using pilot symbol assisted modulation (PSAM). An overview of this project is provided, while its challenges are detailed later. The adopted technical approach is discussed, and the outcome of the project is detailed later.

Project Overview - The USRP has recently been employed as the basis of an undergraduate dissertation project at the University of Southampton, United Kingdom. This project provided one third of the final-year credit for a Bachelor of Engineering undergraduate student, who completed this work on an individual basis between October 2012 and May 2013. The objective of the dissertation project is for the student to demonstrate an ability to work independently on a particular topic. This is achieved by carrying out reading, and other research, design, planning, implementation, and testing. The project also requires the student to demonstrate the ability to explain technical work both verbally and in written form. The student allocated to this dissertation project was tasked with converting the earlier described DPSK transceiver into a PSK one. This required a number of significant additional practical challenges to be overcome, as discussed in the next section.

Practical Challenges - As described earlier, DPSK facilitates non-coherent demodulation, dispensing with channel estimation. However, this is done at the expense of a single transmission error causing two demodulation errors, since the affected DPSK symbol will affect the decoding of both its previous symbol and its next symbol [9].

This motivates PSK, in which digital information is directly modulated onto PSK symbols individually, ensuring that a single transmission error causes only a single demodulation error. This potentially enables PSK to be used for $3 \mathrm{~dB}$ lower channel signal-to-noise ratios (SNRs) than non-coherent DPSK, without degrading the bit error rate (BER) [9]. However, PSK requires coherent demodulation, which requires channel estimation in order to equalize the received signal before it can realize its potential to offer 3 $\mathrm{dB}$ gain over non-coherent DPSK. When implementing a PSK scheme, channel estimation represents a significant practical challenge that must be overcome in addition to those offered by DPSK, as discussed earlier. In contrast, simulation-based PSK projects often assume that perfect channel knowledge is magically available to the receiver without considering how this may be obtained.

Channel estimation can be realized using PSAM [10], which periodically intersperses the transmitted PSK data symbols with pilot symbols, as shown in Fig. 6. These pilot symbols are known to the receiver, allowing the received symbol values to be compared with the known transmitted values in order to estimate the corresponding channel. Interpolation between pairs of consecutive pilot symbols can be employed in order to estimate the channel over all $N$ data symbols. The optimal placement of the pilot symbols [11] depends on the normalized Doppler frequency of the time-selective fading channel, as well as the degree of carrier mismatch between the transmitter and receiver. Therefore, the design and implementation of PSAM represents a significant practical challenge, which is not encountered when implementing the above DPSK scheme.

Note that the receiver of a PSK-modulated system employing PSAM requires a mechanism for distinguishing between data symbols and pilot symbols. This can be achieved using frame synchronization [12], which arranges groups of consecutive data and pilot symbols into frames. 
As shown in Fig. 6, each frame begins with a sequence of synchronization symbols, which are obtained by DPSK modulating a pseudo-random sequence of digital information that is known to both the transmitter and receiver. With this arrangement, the receiver continually attempts non-coherent DPSK demodulation until the pseudo-random synchronization sequence is encountered. Once the synchronization symbols have been identified in this manner, the receiver can exploit knowledge of $N$ to identify the following symbols as periodic patterns of data and pilot symbols and distinguish between them. The design and implementation of a frame synchronization mechanism represents a significant practical challenge, which is typically overlooked in simulation-based PSK projects, by assuming that the transmitter and receiver are magically synchronized perfectly.

Technical Approach - At the University of Southampton, undergraduate dissertation projects are assessed against a number of criteria, including achievement, project management, technical approach, testing, and evaluation. In order to meet these criteria, the student adopted a step-by-step approach to the design, implementation, testing, and characterization of the described PSK scheme employing PSAM.

- The student modified the DPSK transmitter so that it transmitted individual frames comprising only eight DPSK synchronization symbols rather than continually streaming DPSK data symbols. Then a brute-force search was performed on the demodulated digital information in order to locate the pseudo-random synchronization sequence. Testing at this stage comprised ensuring that the synchronization sequence could be reliably received and located when communicating over the air between USRPs separated by several meters.

- The student modified the DPSK transmitter to concatenate the eight DPSK synchronization symbols of each transmission frame with $N=10$ data symbols. However, at this stage, DPSK modulation was employed to generate these data symbols rather than PSK modulation as shown in Fig. 6. The receiver was modified to demodulate the $N=10$ DPSK data symbols separately from the eight DPSK synchronization symbols once the frame had been located as described above. The system was tested by confirming that demodulation errors were reliably avoided for the data symbols.

- The student modified the transmitter and receiver to employ transmission frames comprising 1000 sequences of $N=10$ DPSK-modulated data symbols separated by PSK-modulated pilot symbols. This was tested by confirming that the new transmission frame structure did not prevent the reliable non-coherent DPSK demodulation of the data symbols. Channel estimation was implemented by estimating the channel per pilot and then interpolating between the different pilot channel estimates.

-The PSK scheme using PSAM was completed by employing PSK modulation for the data symbols, as shown in Fig. 6. This was tested by ensuring that the data symbols could be recovered reliably when communicating over the air

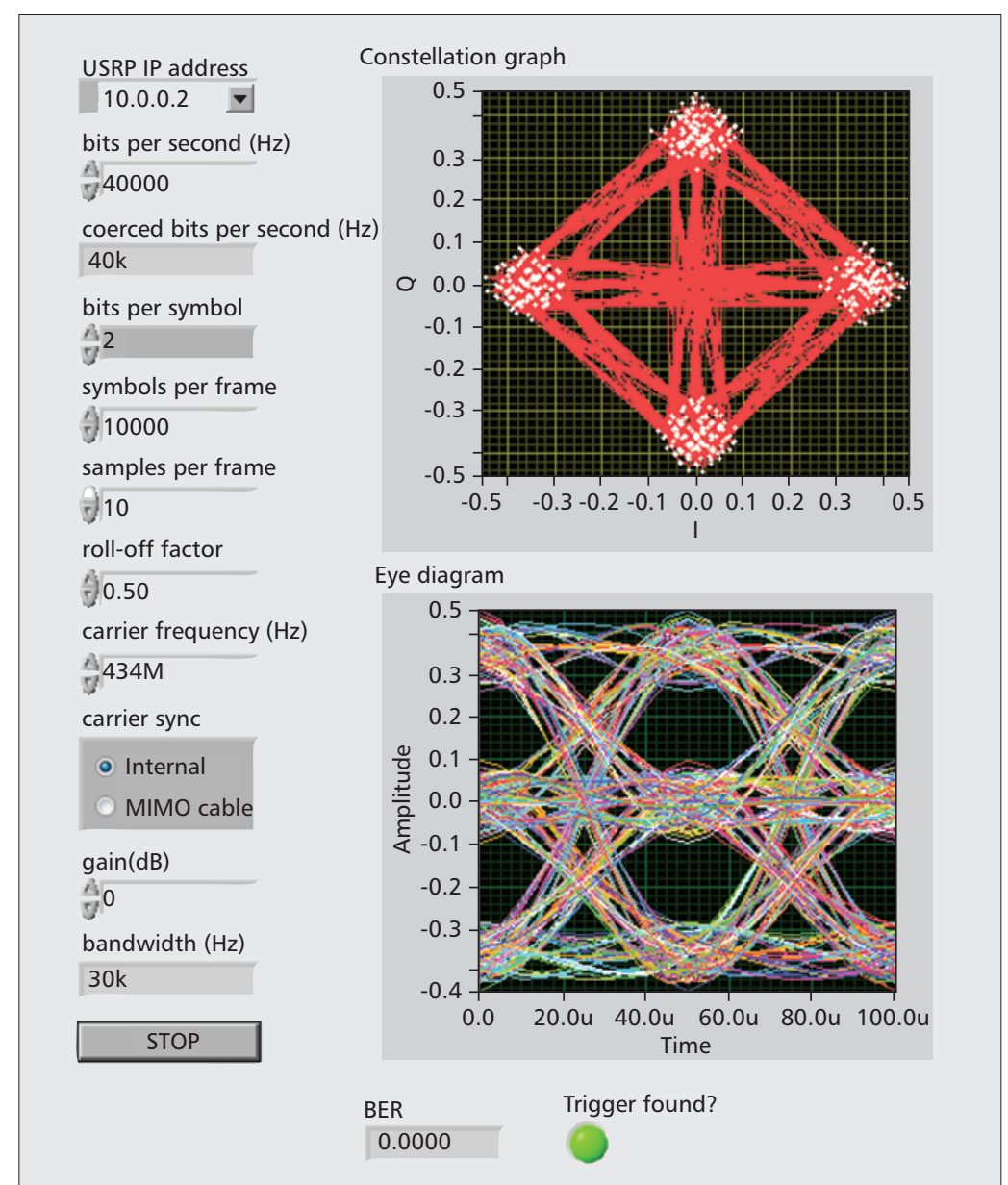

Figure 5. The LabVIEW front panel of the DQPSK scheme, including various input parameters and eye diagrams.

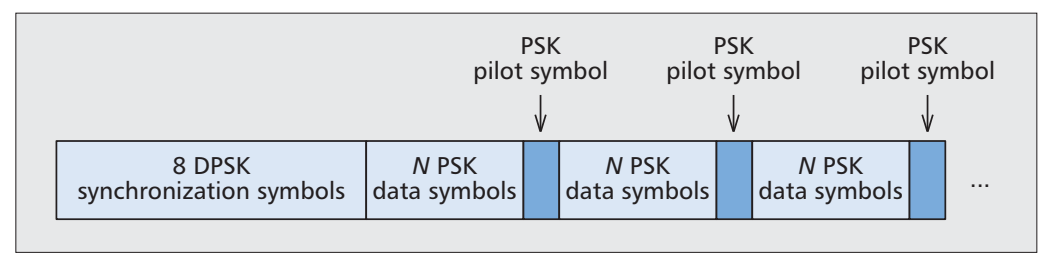

Figure 6. Transmitted frame structure in a PSK scheme using PSAM.

between USRPs separated by several meters.

-In a final step, the BER performance of the developed PSK scheme using PSAM was evaluated by combining the developed transmitter and receiver into a single LabVIEW VI and separating them with a parametrizable model of a time-selective fading channel. This was used to plot the BER of the developed scheme vs. channel SNR as functions of various system parameters, including the PSK modulation order, the number $N$ of data symbols per pilot symbol, as well as the normalized Doppler frequency and the Rician $K$ factor of the time-selective fading channel. These BER plots were validated by comparing them with analytic expressions for the BER [9]. The developed scheme was found to suffer from about $2 \mathrm{~dB}$ loss compared to the analytic expressions, since the theoretical analysis makes the impractical assumption of perfect synchronization and channel estimation. 
it is important to

teach students the importance of testing their VIs as they are building their systems instead of building the whole system and then finding that it does not work and is very difficult to debug.
Project Outcome - The student documented the above approach in a dissertation and presentation. The student was awarded a first-class grade for this project in recognition of the carefully adopted approach to project management, implementation, testing, and evaluation. The student offered the following reflections on this project: "This project has allowed me to enhance my depth of understanding in various aspects of modulation and demodulation in a communications system. It has also enabled me to validate my theoretical knowledge in the real world, by using the USRP for over-the-air transmission. The modulation and demodulation process is clearly represented by LabVIEW, which enabled me to design, test and debug each stage of the process with ease."

\section{REFLECTIONS}

In this section, we offer some reflections drawing on our experiences in supervising projects in which the USRP was employed to demonstrate the practical challenges of wireless communication.

For many of our students, these projects served as their first introduction to LabVIEW. Therefore, before exposing the students to the USRP, we gave them a short lecture and tutorial, as described earlier. This introduced the basics of graphical programming in LabVIEW, but was not intended to give the students sufficient knowledge or experience to start with a blank VI and interface it with the USRP. Hence, rather than devoting several hours of tutoring to this purpose, we opted to give the students "onthe-job" training. More specifically, we gave the students partially completed LabVIEW VIs, in which the program structure and interface with the USRP were already provided. We instructed the students to focus on implementing the various components of the transceiver, and this way the students were able to immediately begin meeting the intended learning outcomes. As the projects progressed, we found that the students were able to gradually acquire knowledge and experience in LabVIEW's program structure and interfacing with the USRP.

Furthermore, we found that for some students, the natural inclination was to attempt to implement the entire wireless communication scheme before testing it. This means that when they attempted to test their system and it did not work, the debugging task to locate the programming error would be very difficult. In order to mitigate this, we carefully structured the projects so that they invited the students to design, implement, test, and debug their systems in a step-bystep approach, as exemplified in the previous section. By ensuring that each stage of the scheme operates as expected before moving on to the next, the search for programming errors can be significantly simplified and restricted to the most recently implemented stage. We found that by adopting this approach, our students enjoyed a significantly increased probability of successfully implementing a fully operational wireless communication scheme.

We plan to continue expanding our employ- ment of the USRP in both undergraduate and postgraduate student projects on further practical challenges of wireless communications. Possible projects include implementing coherent modulation using higher order constellations such as 16-quadrature amplitude modulation (QAM), MIMO techniques, code-division multiple access (CDMA), orthogonal frequency-division multiplexing (OFDM), and channel coding including convolutional codes as well as nearcapacity codes such as turbo codes. Finally, note that this is not a comprehensive list of potential projects, but these will expose students to most of the challenges experienced when designing the communications systems included previously.

\section{CONCLUSION}

In this article, we have presented how the USRP can be used to give students hands-on experiences and teach them how to link theory with practical implementation instead of only using theoretical analysis and simulations. Additionally, we used the USRP in order for the students to appreciate the challenges in designing communications systems and learn how to overcome these challenges. Most students appreciated using the USRP and wanted to make more use of it. On the other hand, we learned from the USRP projects that teaching students LabVIEW using practical sessions and making the software available for them to use in the university or on their own PCs are essential for students to learn the software quickly and use it to build their systems. Additionally, it is important to teach students the importance of testing their VIs as they are building their systems instead of building the whole system and then finding that it does not work and is very difficult to debug.

\section{REFERENCES}

[1] S. Cass, "Tools Toys: Hardware for your Software Radio," IEEE Spectrum, vol. 43, no. 10, 2006, pp. 51-54.

[2] L. Hanzo et al., Near-Capacity Variable-Length Coding: Regular and EXIT-Chart- Aided Irregular Designs, WileyIEEE Press, Nov. 2010

[3] J. Proakis and M. Salehi, Digital Communications, 5th ed., McGraw Hill, 2010.

[4] C.R. Johnson, Jr. and W. A. Sethares, Telecommunication Breakdown: Concepts of Communications Transmitted via Software-Defined Radio, Prentice Hall, 2003.

[5] C. R. Rojas, P. Zetterberg, and P. Handel, "Transceiver Inphase/Quadrature Imbalance, Ellipse Fitting, and the Universal Software Radio Peripheral," IEEE Trans.Instrumentation and Measurement, vol. 60, no. 11, 2011, pp. 3629-39.

[6] T.B. Welch and S. Shearman, "Teaching Software Defined Radio Using the USRP and LabVIEW," IEEE Int'I. Conf. Acoustics, Speech and Signal Proc., 2012, pp. 2789-92.

[7] Univ. Southampton, Master of Science in Wireless Communications, http://www.ecs.soton.ac.uk/programmes/ msc-wirelesscommunications.

[8] L. Hanzo et al., Quadrature Amplitude Modulation: From Basics to Adaptive Trellis-Coded, Turbo-Equalised and Space-Time Coded OFDM, CDMA and MC-CDMA Systems, 2nd ed., Wiley, 2004.

[9] C. K. Pauw and D. L. Schilling "Probability of Error of $M$-ary PSK and DPSK on A Rayleigh Fading Channel," IEEE Trans. Commun., vol. 36, June 1988, pp. 755-56.

[10] J. K. Cavers, "An Analysis of Pilot Symbol Assisted Modulation for Rayleigh Fading Channels," IEEE Trans. Vehic. Tech., vol. 40, Nov. 1991, pp. 686-93.

[11] M. Dong, L. Tong and B.M. Sadler, "Optimal Insertion of Pilot Symbols for Transmissions over Time-Varying Flat Fading Channels," IEEE Trans. Signal Proc., vol. 52, May 2004, pp. 1403-18.

[12] J. A. Gansman, M. P. Fitz and J. V. Krogmeier, "Opti- 
mum and Suboptimum Frame Synchronization for Pilot-Symbol-Assisted Modulation," IEEE Trans. Commun., vol. 45, Oct.1997, pp. 1327-37.

\section{BIOGRAPHIES}

MOHAMmed EL-HAJJAR (meh@ecs.soton.ac.uk) is a lecturer in the Department of Electronics and Computer Science at the University of Southampton. He received his B.Eng. degree in electrical engineering from the American University of Beirut, Lebanon, in 2004. He then received an M.Sc. in radio frequency communication systems and a Ph.D. in wireless communications, both from the University of Southampton, United Kingdom, in 2005 and 2008, respectively. Following his Ph.D., he joined Imagination Technologies as a design engineer, where he worked on designing and developing the BICM peripherals in Imagination's multi-standard communications platform, which resulted in several patents. In January 2012, he joined the Electronics and Computer Science Department in the University of Southampton as a lecturer in the Communications, Signal Processing and Control research group. He is the recipient of several academic awards, and has published a WileyIEEE book and in excess of 45 journal and international conference papers. His research interests are mainly in the development of intelligent communications systems for the Internet of Things, including energy-efficient transceiver design, cross-layer optimization for large-scale networks, massive MIMO systems for millimeter-wave communications, cooperative communications, and radio over fiber systems.

Quoc A. NGUYEN has studied in the Department of Electronics and Computer Science, University of Southampton since October 2010. He was awarded a first class honours B.Eng in electronic engineering in July 2013. He now works at Samsung Vietnam Mobile R\&D Center in Hanoi.

ROBERT G. MAUNDER [SM](rm@ecs.soton.ac.uk) joined the Department of Electronics and Computer Science, University of Southampton, in October 2000. He was awarded a first class honours B.Eng. in electronic engineering in July 2003, as well as a Ph.D. in wireless communications and a lectureship in December 2007. In 2013, he was promoted to senior lecturer. He is a Chartered Engineer of the IET. His research interests include joint source/channel coding, iterative decoding, irregular coding, and modulation techniques. He has published a number of IEEE papers in these areas.

Soon XIN NG [S'99, M’03, SM'08] (sxn@ecs.soton.ac.uk) received his B.Eng. degree (first class) in electronics engineering and his Ph.D. degree in wireless communications from the University of Southampton in 1999 and 2002, respectively. From 2003 to 2006, he was a postdoctoral research fellow working on collaborative European research projects known as SCOUT, NEWCOM, and PHOENIX. Since August 2006, he has been a member of academic staff in the School of Electronics and Computer Science, University of Southampton. He is involved in the OPTIMIX and CONCERTO European projects as well as the IU-ATC and UC4G projects. He is currently a senior lecturer at the University of Southampton. His research interests include adaptive coded modulation, coded modulation, channel coding, space-time coding, joint source and channel coding, iterative detection, OFDM, MIMO, cooperative communications, distributed coding, quantum error correction codes, and joint wireless and optical fiber communications. He has published over 160 papers and co-authored two Wiley/IEEE Press books in this field. He is a Chartered Engineer and a Fellow of the Higher Education Academy in the United Kingdom. 